\title{
Mitophagy in Traumatic Brain Injury: A New Target for Therapeutic Intervention
}

\author{
Mingrui Zhu, ${ }^{1}$ Xinqi Huang, ${ }^{1}$ Haiyan Shan $\mathbb{D}^{2}$, and Mingyang Zhang $\mathbb{D}^{1}$ \\ ${ }^{1}$ Institute of Forensic Sciences, School of Basic Medicine and Biological Sciences, Soochow University, Suzhou, China \\ ${ }^{2}$ Department of Obstetrics and Gynecology, The Affiliated Suzhou Hospital of Nanjing Medical University, Suzhou, China \\ Correspondence should be addressed to Haiyan Shan; ghostqth@163.com and Mingyang Zhang; ghost8469@163.com
}

Received 13 September 2021; Revised 31 December 2021; Accepted 10 January 2022; Published 27 January 2022

Academic Editor: Athanasios Alexiou

Copyright ( 2022 Mingrui Zhu et al. This is an open access article distributed under the Creative Commons Attribution License, which permits unrestricted use, distribution, and reproduction in any medium, provided the original work is properly cited.

\begin{abstract}
Traumatic brain injury (TBI) contributes to death, and disability worldwide more than any other traumatic insult and damage to cellular components including mitochondria leads to the impairment of cellular functions and brain function. In neurons, mitophagy, autophagy-mediated degradation of damaged mitochondria, is a key process in cellular quality control including mitochondrial homeostasis and energy supply and plays a fundamental role in neuronal survival and health. Conversely, defective mitophagy leads to the accumulation of damaged mitochondria and cellular dysfunction, contributing to inflammation, oxidative stress, and neuronal cell death. Therefore, an extensive characterization of mitophagy-related protective mechanisms, taking into account the complex mechanisms by which each molecular player is connected to the others, may provide a rationale for the development of new therapeutic strategies in TBI patients. Here, we discuss the contribution of defective mitophagy in TBI, and the underlying molecular mechanisms of mitophagy in inflammation, oxidative stress, and neuronal cell death highlight novel therapeutics based on newly discovered mitophagy-inducing strategies.
\end{abstract}

\section{Introduction}

Mitochondria are organelles coated by bilayer membranes, mitochondrial inner membrane (IMM), and mitochondrial outer membrane (OMM), which are the main places for cellular respiration and energy production [1]. The cristae, folded in the inner membrane, is a very important structure studded with ATP synthase and a variety of cytochromes $[2,3]$. The brain consumes approximately $20 \%$ of the total body energy even though it accounts for only $2 \%$ of the body weight [4]. This huge energy consumption depends on the utilization of glucose and the maintenance of mitochondrial function [5]. Therefore, the maintenance of mitochondrial network integrity and activity is a prerequisite for nervous system homeostasis [6]. Defective mitochondria can be toxic by generating excessive amounts of ROS, by consuming ATP through the reversal of ATP synthase, and by interfering with a host of other metabolic processes [7]. Mitophagy is a crucial process of eliminating old or damaged mitochondria for the maintenance of the integrity of the mitochondrial pool for cellular homeostasis, which impacts various physiological and pathological courses in the brain. Recent advances in the field of brain research have revealed the pivotal role of mitophagy in neuronal cell fate and neurological function $[8,9]$. However, the potential of targeting mitophagy as a therapeutic strategy in traumatic brain injury (TBI) remains to be explored. Moreover, several proteins including Cardiolipin, Parkin, and BNIP3L/NIX, which are responsible for mitophagy, have been revealed to be deregulated in TBI $[10,11]$. This review is aimed at describing the research progress of mitophagy including the molecular pathways that govern mitophagy and the role of mitophagy in different aspects of TBI and other brain diseases, to develop potential therapeutics for clinical treatment (Figure 1).

\section{The Mitophagy Pathways in TBI}

Mitophagy is the targeted phagocytosis and destruction of damaged mitochondria by autophagy, whose function is to strictly control the quality and quantity of mitochondria 


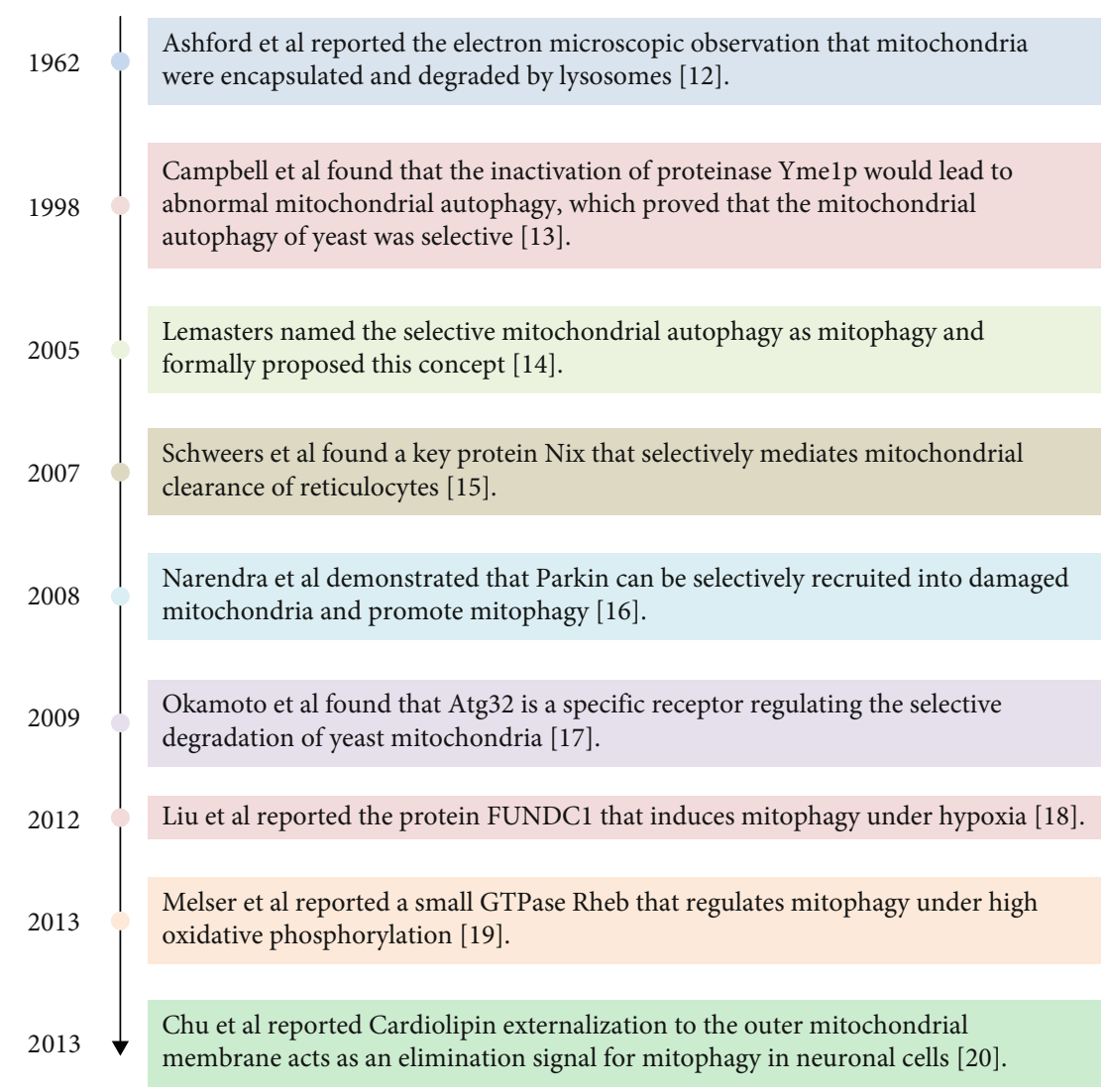

Figure 1: Discovery process of mitophagy [12-20].

[21]. Mitophagy is activated by a series of pathways, such as ubiquitin-mediated pathway [22] and mitochondrial receptor-mediated pathway $[23,24]$. The possible mitophagy pathways in TBI are summarized in Figure 2.

Due to an energy crisis emerging post-TBI as the increased energetic demand cannot be met, the level of OXPHOS increased, and ROS levels exceeded a certain threshold. Meanwhile, hypoxia can be initiated by TBIinduced cerebral hypoperfusion. Decrease of mitochondrial membrane potential $(\triangle \Psi m)$ was also found in the brain after TBI. All the events mentioned above induce mitochondrial damage and activate different mitophagy pathways. The change in energy state caused by OXPHOS will activate Rheb pathway. Decreased mitochondrial membrane potential increases membrane depolarization and the accumulation of PINK1 kinase in the OMM. Activated PINK1/ Parkin at the OMM allows the interaction with LC3 for the autophagic degradation through the specific adaptor proteins, such as p62, which binds at the same time with ubiquitin and LC3. At this time, PINK1 can recruit and activate Parkin, which regulates mitophagy by ubiquitinating Mfn2, Miro1, and VDAC. The other mechanism of mitophagy induced by stress, such as ROS and hypoxia, is regulated by mitochondrial receptors such as BNIP3, BNIP3L/NIX, FUNDC1, and Cardiolipin (CL) which directly bind with LC3 through the conserved LC3 interacting region (LIR) motif. After the above mitophagy pathways are activated, damaged mitochondria are selectively elimi- nated, the quality of mitochondria is guaranteed, and neuronal cells are homeostasis maintained, which improves neurological function recovery in TBI.

2.1. PINK1/Parkin-Mediated Ubiquitin Pathway. PTENinduced putative kinase 1 (PINK1) is a serine/threonine protein kinase, and Parkin is a member of the E3 ubiquitin ligase complex [25]. When the mitochondrial membrane potential decreases, the expression of PINK1 is upregulated, aggregates on the mitochondrial membrane, and then, phosphorylates Ser65 in the ubiquitin-like protein domain of parkin, leading to conformational changes in the tertiary structure for the activation of parkin [26]. Activated PINK1/Parkin at the OMM allows the interaction with LC3 for autophagic degradation through specific adaptor proteins such as p62, OPTN, NDP52, and TAXBP1 which bind at the same time with ubiquitin and LC3 [16]. Our laboratory reported that the expression levels of PINK1 and Parkin were increased at $24 \mathrm{~h}$ after TBI and Mdivi-1 inhibited the activation of the PINK1 and Parkin pathway, suggesting that PINK1/Parkin-mediated mitophagy was activated in TBI [27]. Chen et al. demonstrated that PGAM5 deficiency blocked Parkin and PINK1 translocation to mitochondria and alleviated neuroinflammation in TBI mouse model [28]. Ren et al. found that the expression of PINK1 was significantly induced by TBI, and RvD1 treatment successfully downregulated it [29]. These results suggested that downregulation of PINK1/Parkin-mediated mitophagy might play an important role in recovery after 


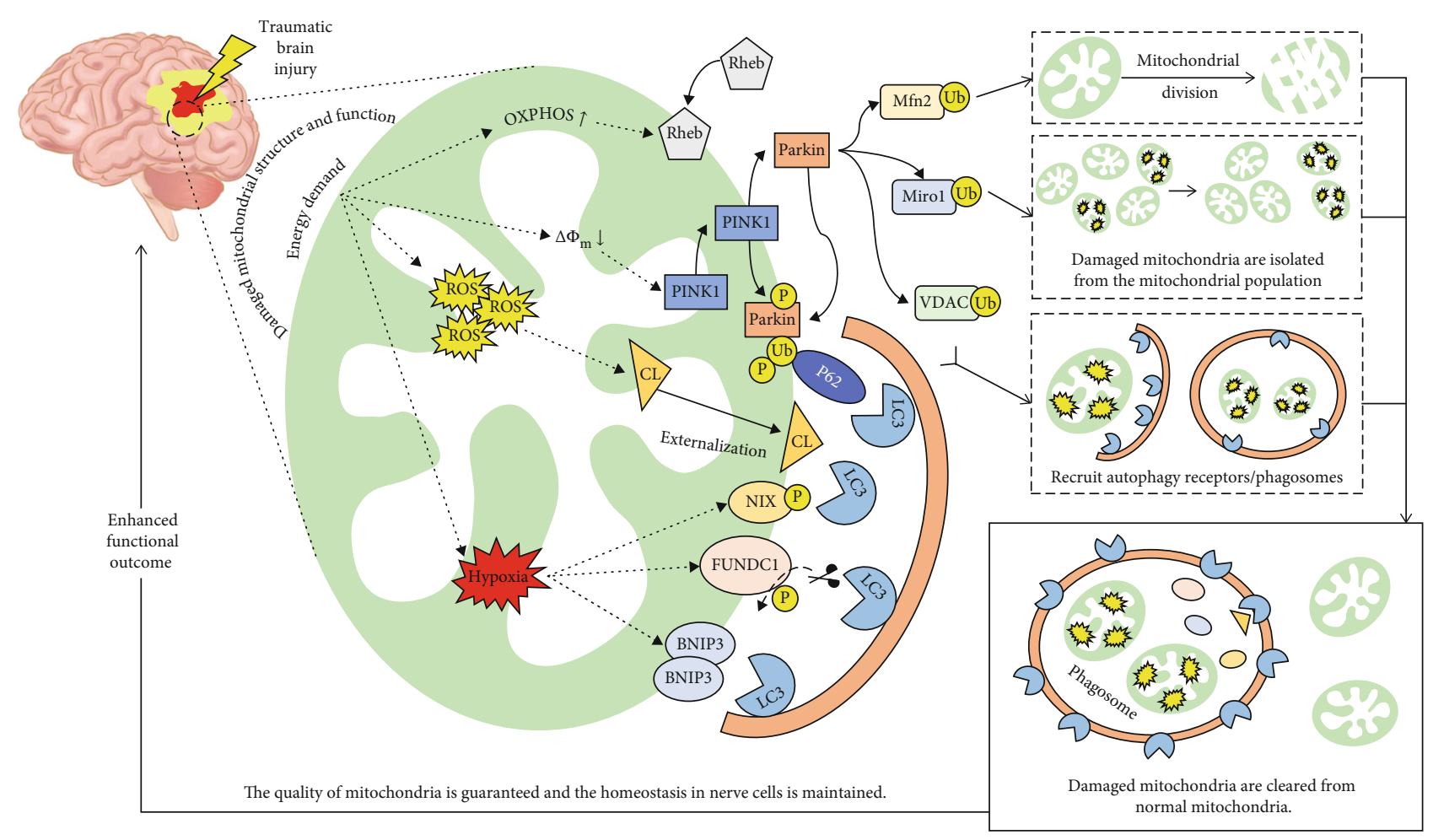

FIgUre 2: The mitophagy pathways in TBI.

TBI. However, Lin et al. found that PINK1 knockout mice suffering from TBI demonstrated little recovery after thyroid hormone treatment, suggesting the involvement of PINK1mediated mitophagy in neurogenesis and neuroprotection [30]. The exact role of PINK1/Parkin-mediated mitophagy in traumatic brain injury is still unclear. The decreased expression of PINK1 and Parkin may be caused by the improvement of mitochondrial membrane potential, which is only the feedback result for mitochondrial function recovery after drug treatment and is not the reason for the improvement of brain function. PINK1/Parkin pathway alterations have also been found in many neurodegenerative diseases such as Parkinson's disease (PD), Alzheimer's disease (AD), and Huntington's disease (HD). Parkin overexpression can rescue the mitochondrial dysfunction in $\mathrm{AD}$ [8]. However, downregulating expression of either PINK1 or Parkin genes ameliorated neurodegeneration phenotypes in amyotrophic lateral sclerosis (ALS) [31]. Wen et al. reported that PINK1 overexpression-mediated parkin regulation is key to the protection against cerebral ischemia-reperfusion injury [32]. Therefore, we need many experiments to verify this exact role of PINK1/Parkin in TBI.

2.2. Receptor-Mediated Pathway. The other mechanism of mitophagy in a context-dependent manner is regulated by the mitochondrial receptors like BNIP3, BNIP3L/NIX, FUNDC1, NIPSNAP1, NIPSNAP2, BCL2L13, PHB2, FKBP8, Rheb, and Cardiolipin, which directly binds with LC3 through the conserved LC3 interacting region (LIR) motif. BCL2/adenovirus E1B $19 \mathrm{kDa}$ interacting protein 3 (BNIP3), NIP3-like protein X (BNIP3L/NIX), BCL2-like 13
(BCL2L13), and FUN14 domain containing 1 (FUNDC1) are marker proteins on the OMM. They all interact with Atg8 protein family members on phagosomes through conserved LC3 interacting region (LIR) as mitochondrial receptors under hypoxia, so as to recruit phagosomes and clear mitochondria damaged by hypoxia [33]. Nitrophenylphosphatase domain and nonneuronal SNAP25-like protein homolog 1 (NIPSNAP1) and NIPSNAP2, Prohibitin 2 (PHB2), FKBP prolyl isomerase 8 (FKBP8/FKBP38) FKBP8, and Cardiolipin (CL) are located in the IMM [34]. In many cases, the IMM protein requires the rupture of the OMM to recruit the mitophagy molecular machinery. However, in some cases, this dynamic positional change of the IMM protein is not necessary for mitophagy [35]. Alternatively, they may act as direct autophagy receptors in the IMM and binds to LC3 through the classical LIR motif. There have been few reports on the role of the mitochondrial receptor-mediated mitophagy pathway in TBI. Ma et al. found that the expression of NIX decreased after TBI and NIX overexpression plays a neuroprotective role in TBI-induced damage through autophagy and apoptosis pathways [10]. In intracerebral hemorrhage and ischemia-reperfusion brain injury, NIX protein levels are significantly elevated and overexpression of NIX could be a potential therapeutic target for hemorrhagic and ischemic stroke $[36,37]$. There is no doubt that hypoxia is the basic pathological mechanism in many brain diseases, including cerebral ischemic injury, TBI, and cerebral hemorrhage. However, the changes in NIX expression levels after injury in brain diseases are different, suggesting that NIX-mediated mitophagy is related not only to hypoxia but also to other pathophysiological processes, such as the 
cell death pathway $[38,39]$. Mitochondrial lipid signaling is a hot field in TBI research. Cardiolipin (CL) is one of the unique lipids in mitochondrial intima, accounting for 15$20 \%$ of all mitochondrial lipids [40]. Kalgudi et al. found that free circulating Cardiolipins in the plasma released from the injured brain with a disrupted BBB after TBI and Cardiolipin were detectable in approximately $18 \%$ of patients after severe TBI, confirming that circulating Cardiolipin plays an important role in the pathogenesis of severe TBI in humans $[41,42]$. In the TBI model, it can be observed that the selective oxidation of brain Cardiolipin begins 3 hours after injury, and enrichment and collapse of the CL were found in the IMM $[11,43]$. At this time, externalized Cardiolipin can directly interact with LC3 and then promote the occurrence of mitophagy [21]. Chao et al. demonstrated that Cardiolipin-dependent mitophagy is activated by TBI, and suppression of TBI-induced mitophagy worsens the overall outcome [11]. For the Cardiolipin pathway, mitophagy can be inhibited by reducing the content of mitochondrial Cardiolipin or the expression of phospholipid scramblases (PLS). In addition, mutagenesis of amino acid residues of LC3 at R10 and R11 can reduce the ability of LC3 peptide to bind Cardiolipin in vitro and weaken the recruitment of phagosomes by LC3 in living cells [44]. Cardiolipin exposure to the outer membrane not only affects mitophagy but also modulates $\alpha$-synuclein aggregation and affects the electron transport in PD [45]. In contrast to the above pathways, Rheb is mainly located in the OMM and matrix, which regulates mitophagy related to energy state and ensures the efficiency of mitochondrial energy production [46-48]. Our group has recently revealed that Rheb is recruited to damage mitochondria for their engulfment within mitophagosomes in the axons of neurons, and such a mechanism promotes mitophagic removal of stressed mitochondria from distal axons to maintain mitochondrial homeostasis $[49,50]$. However, no reports thus far have studied the effects of mitochondrial receptors other than BNIP3L/NIX and Cardiolipin on mitophagy in TBI models. Therefore, this is an interesting aspect worth exploring.

\section{The Role of Mitophagy in TBI}

TBI is a traumatic structural injury and brain dysfunction caused by external forces [51], which usually originates from the primary injury caused by external causes, and then, gradually develops into secondary injury related to inflammatory response, oxidative stress, and cell death. In the occurrence and development of TBI, these biological processes interact with mitophagy to regulate mitochondrial quality, which could affect the survival and death of nerve cells (Figure 3).

Traumatic brain injury results in more severe oxidative damage, inflammatory response, and cell death, which are associated with mitochondrial dysfunction. Damage to mitochondria will release a large amount of reactive oxygen species and promote the release of proinflammatory factors, which lead to oxidative stress damage and inflammation. At the same time, damaged mitochondria result in the activation of mitophagy. If mitophagy is not enough to activate, the imbalance of the delicate equilibrium among mitophagy, ROS production, and inflammatory response can start, drive, or accelerate the cell death process. Therapeutic targets that upregulate mitophagy to reduce downstream cascades such as oxidative damage, inflammatory response, and cell death may improve neurological dysfunction after TBI.

3.1. Inflammation and Mitophagy. Recently, mitochondrial damage caused by TBI has gradually attracted attention, and the improvement of brain mitochondrial function has become a new target for the treatment of TBI. In the past, the main role of drugs for the treatment of TBI was to inhibit posttraumatic inflammatory response [52]. However, some drugs not only prevent inflammation induced by TBI but also improve mitochondrial function by the mitophagy pathway. Ren et al. found that Resolvin D1 not only inhibits neuroinflammation but also eliminates damaged mitochondria through mitophagy [29]. Similarly, IL-10 and p53 activators pifithrin- $\mu$ and pifithrin- $\alpha$ can also provide neuroprotection by regulating neuroinflammation and mitophagy [53, 54]. In addition, it was also found that rapamycin-induced mitophagy could work together with the inhibition of the activation of NLRP3-mediated inflammatory bodies, and the combined treatment had better neuroprotective effect [55]. Moreover, Lin et al. found that the inhibitory effect of melatonin on inflammation is regulated by activating mitophagy and selectively clearing damaged mitochondria. Inhibiting mitophagy will significantly enhance the inflammation caused by TBI, as direct evidence links mitophagy to inflammation [56]. Chen et al. demonstrated that PINK1-mediated mitophagy and the NLRP3 inflammasome have the interactivity, and mitophagy further enhances the neuroprotection by inhibiting NLRP3 inflammasome activation post-TBI [55]. Lin et al. highlight a role for melatonin in protecting against TBI-triggered immunopathology, which is accomplished by negatively regulating inflammation activation via mitophagy [56]. Ding et al. showed the fisetin-blocked inflammation activation via promoting mitophagy in sepsis-associated encephalopathy [57]. Zheng et al. reported that FUNDC1, as a mitophagy receptor, alleviated ICH-induced inflammation by promoting mitophagy, suggesting that FUNDC1 might be a new therapeutic target for ICH treatment [58]. Taken together, these data demonstrate that inflammation can cause accumulation of damaged mitochondria, and impaired mitophagy of these mitochondria results in the promotion of proinflammatory pathways. Thus, therapeutic targets that upregulate mitophagy to reduce downstream cascades induced by damaged mitochondria may improve inflammation.

3.2. Oxidative Stress and Mitophagy. During TBI, the increased energy demand leads to excessive ROS production, resulting in mitochondrial DNA damage and dysfunction [21]. Mitophagy is an endogenous neuroprotective process for targeted damaged mitochondria, and then, the damaged mitochondria can be cleared in time, which is necessary to maintain the normal function of neurons [59]. In addition, mitophagy can also reduce gastrointestinal dysfunction caused by TBI. Liu et al. found that mitophagy can reduce 


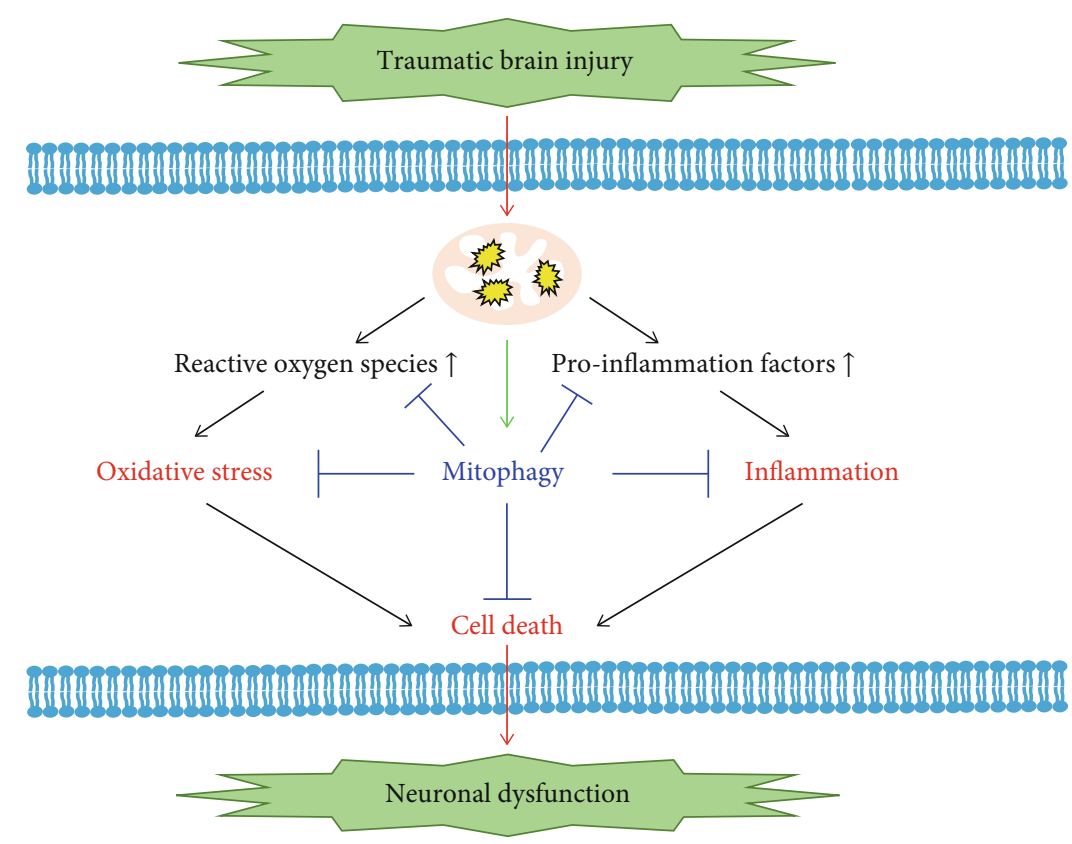

FIgUre 3: The role of mitophagy in TBI.

intestinal mucosal injury and epithelial barrier dysfunction by reducing oxidative stress after TBI [60]. However, the activation degree of mitophagy is also different due to traumatic environments and stimulation conditions. Moderate mitophagy can target damaged mitochondria, which maintains the function of mitochondria and is beneficial to the survival of neurons [61]. When mitophagy is insufficient, the damaged mitochondria are not properly cleared, and ROS continue to accumulate, resulting in the oxidation of mitochondrial proteins and DNA and the aggravation of mitochondrial damage [62]. Excessive mitophagy removes too many mitochondria [44]. Because mitochondria are crucial to neurons, excessive mitochondrial clearance will promote nerve cell death and further affect brain function [63]. Wu et al. found that mitochondrial division inhibitor 1 (Mdivi-1) can reduce TBI-induced blood-brain barrier damage and cell death by inhibiting mitophagy while inhibiting mitochondrial division [27]. In contrast, Niu et al. found that Mdivi-1 could aggravate the damage of TBI rats, indicating that mitophagy played a protective role on damaged mitochondria [64]. As noted above, the results may be caused by the degree of mitophagy. Therefore, activation of mild mitophagy or inhibition of excessive mitophagy may have beneficial effects on the structure and function of mitochondria after TBI and even on the survival of neurons [65]. In addition, it appears that mitophagy pathways differ spatially and kinetically in neurons and immortalized cells and therefore might diverge in their ultimate outcome and function. Mitophagy in nerve cells has a higher threshold than other cells and tends to local repair mechanisms or partial degradation mechanisms [44]. In 2020, Zakarya et al. found that 5,6-dicarboxy-1,1,3,3-tetraethylisoindolin-2-yloxyl (DCTEIO), as a superoxide dismutase simulant, can maintain the structure and function of mitochondria by scavenging ROS, reduce oxidative stress, promote mitophagy, and then, improve the tissue repair and neural function of TBI rats. Animal experiments also showed that an obvious increase in mitophagy, and the damage range of brain tissue decreased at 24 hours and 6 weeks after injury when treated with DCTEIO immediately after injury [66]. Lin et al. demonstrated that triiodothyronine (T3) promoted the clearance of damaged mitochondria by promoting PINK1 and significantly reduced the production of ROS, indicating that T3 reduced neuronal death through mitophagy [30]. These results indicate that mitophagy prevents the accumulation of damaged mitochondria and ROS.

3.3. Cell Death and Mitophagy. Cell death is the final solution for a neuron only when multiple stresses are piled up to a level beyond cell's recovery capacity [67]. A traumatic incident, such as TBI, causes a sudden decline of energy production in the affected neurons and elicits acute neuronal cell death, which is commonly seen in neurodegenerative diseases. Two waves of neuronal cell death, necrosis, and programmed cell death occur after TBI. Early application of neuroprotective protocols seems critical for any possibility of reducing neuronal necrosis induced by membrane disruption and irreversible metabolic disturbances. This second wave of neuronal programmed cell death presents within a time window that may be responsive to targeted therapies $[68,69]$. Recent studies have shown that in addition to necrosis, there are also other new programmed death modes, such as apoptosis, necroptosis, pyroptosis, ferroptosis, entotic cell death, netotic cell death, parthanatos, lysosomedependent cell death, autophagic cell death, alkaliptosis, and oxeiptosis [70]. Mitophagy, the removal of damaged or unwanted mitochondria, was found to be essential for maintaining cellular fitness [71]. Previous work by our group demonstrated the role of mitophagy in regulating cell death [72]. The current review summarizes the relationship 
TABLE 1: Summary of therapeutic development targeting mitophagy in TBI.

\begin{tabular}{|c|c|c|c|c|c|c|}
\hline $\begin{array}{l}\text { Methods or } \\
\text { compounds }\end{array}$ & Time & $\begin{array}{l}\text { Effect on } \\
\text { mitophagy }\end{array}$ & Doses & Action site & Function in TBI & Reference \\
\hline Resolvin D1 & 2020 & Activate & $15 \mu \mathrm{g} / \mathrm{kg}$ & Inflammation & $\begin{array}{l}\text { Ameliorate brain oedema and cognitive impairment, } \\
\text { suppress neuroinflammation and neuronal loss囚eliminate } \\
\text { extra mitoROS, improve the supportive function of } \\
\text { astrocytes }\end{array}$ & [29] \\
\hline IL-10 & 2019 & Activate & Overexpress & Inflammation & $\begin{array}{c}\text { Inhibit inflammatory response, reduce neuronal } \\
\text { degeneration and death }\end{array}$ & [53] \\
\hline $\begin{array}{l}\text { Pifithrin- } \mu \\
\text { Pifithrin- } \alpha\end{array}$ & 2019 & Mitigate & $2 \mathrm{mg} / \mathrm{kg}$ & Inflammation & $\begin{array}{c}\text { Ameliorate neurological functional deficits, attenuate } \\
\text { neuroinflammation, attenuate oxidative stress }\end{array}$ & {$[54]$} \\
\hline Rapamycin & 2019 & Activate & $3 \mathrm{mg} / \mathrm{kg}$ & $\begin{array}{l}\text { Inflammation } \\
\text { Cell death }\end{array}$ & $\begin{array}{c}\text { Attenuate neuroinflammation, mitochondrial damage, } \\
\text { demonstrate neuroprotective effects, inhibit the activation } \\
\text { of NLRP3 inflammasome }\end{array}$ & {$[55]$} \\
\hline Melatonin & 2016 & Activate & $5 \mathrm{ml} / \mathrm{kg}$ & Inflammation & $\begin{array}{c}\text { Repress inflammation, ameliorate neuronal death and } \\
\text { behavioral deficits, dampen the secretion of pro- } \\
\text { inflammatory cytokines }\end{array}$ & {$[56]$} \\
\hline Rapamycin & 2016 & Activate & l & $\begin{array}{l}\text { Oxidative } \\
\text { stress }\end{array}$ & $\begin{array}{l}\text { Alleviate TBI-induced intestinal mucosa damage and } \\
\text { epithelial barrier dysfunction }\end{array}$ & {$[60]$} \\
\hline Mdivi-1 & 2017 & Suppress & $3 \mathrm{mg} / \mathrm{kg}$ & $\begin{array}{l}\text { Oxidative } \\
\text { stress } \\
\text { Cell death }\end{array}$ & $\begin{array}{l}\text { Alleviate loss of mitochondrial membrane potential, ROS } \\
\text { production, ATP reduction, blood-brain barrier disruption } \\
\text { and cell death }\end{array}$ & [27] \\
\hline Mdivi-1 & 2018 & Suppress & $1 \mathrm{mg} / \mathrm{kg}$ & $\begin{array}{l}\text { Oxidative } \\
\text { stress } \\
\text { Cell death }\end{array}$ & $\begin{array}{l}\text { Aggravate neurological manifestations and neuronal } \\
\text { apoptosis }\end{array}$ & {$[64]$} \\
\hline DCTEIO & 2020 & Activate & $\begin{array}{c}40 \mathrm{mM} \\
\text { aqueous } \\
\text { solutions }\end{array}$ & $\begin{array}{l}\text { Oxidative } \\
\text { stress }\end{array}$ & $\begin{array}{c}\text { Scavenge ROS, improve tissue repair and preserve } \\
\text { neurological function }\end{array}$ & [66] \\
\hline Triiodothyronine & 2020 & Activate & $20 \mu \mathrm{g} / 100 \mathrm{~g}$ & $\begin{array}{l}\text { Oxidative } \\
\text { stress } \\
\text { Cell death }\end{array}$ & $\begin{array}{l}\text { Reduce ROS production, prevent neuronal death, induce } \\
\text { neurogenesis and neuroprotection }\end{array}$ & {$[30]$} \\
\hline $\begin{array}{l}\text { Cardiolipin } \\
\text { siRNA }\end{array}$ & 2019 & Activate & $30 \mathrm{nmol}$ & Cell death & $\begin{array}{l}\text { Induce endogenous neuroprotection, limit neuronal } \\
\text { apoptosis and behavioral deficits }\end{array}$ & {$[11]$} \\
\hline
\end{tabular}

between mitophagy and cell death after TBI, as well as mitophagy as a potential therapeutic intervention for altering cell death to improve neurological outcomes. Apoptosis is a type of programmed cell death (PCD). The cytomorphological features of apoptotic cells include shrinkage, chromosome condensation, and DNA fragmentation [67]. Niu et al. demonstrated that Mdivi-1 blocked the induction of mitophagy specifically and activated the apoptosis markers caspase- 3 and caspase- 9 , implying that mitophagy markedly decreased cell apoptosis induced by TBI [64]. Wu et al. reported that Mdivi-1 also alleviated the number of LC3 puncta and TUNEL-positive structures in cells, indicating that autophagy may be involved in the antiapoptotic effects of Mdivi-1 in TBI [27]. Chao et al. showed that TBIinduced Cardiolipin-dependent mitophagy is an endogenous neuroprotective process that limits neuronal apoptosis and behavioral deficits [11]. Lin et al. found that T3 treatment could provide a therapeutic approach for TBI by preventing apoptosis via pink1-mediated mitophagy [30]. Pyroptosis represents a form of programmed cell death that is triggered by proinflammatory signals and associated with inflammation [73]. Recent studies have shown that oxygen deprivation or hypercapnia promotes microglial pyroptosis by inhibiting mitophagy, suggesting that mitophagy acts as a negative regulator of pyroptosis $[74,75]$. Chen et al. demonstrated that rapamycin-induced mitophagy enhances the neuroprotection of inhibition of pyroptosis activation postTBI [55]. Quercetin also prevents neuronal injury via inhibition of pyroptosis activation in microglia by promoting mitophagy in both depression and PD mouse models [76]. Ferroptosis is a new type of cell death that was discovered in recent years and is usually accompanied by a large amount of iron accumulation and lipid peroxidation during the cell death process [77]. It has been reported that ferritin deficiency promotes osteoblastic ferroptosis via the PINK1/ Parkin-mediated mitophagy pathway in type 2 diabetic osteoporosis [78]. However, there is no report about the relationship between mitophagy and other cell deaths including ferroptosis in the brain, and a deeper understanding of the relationship between ferroptosis and multiple diseases may provide new strategies for drug development based on ferroptosis.

3.4. Related Therapeutic Agents Targeting Mitophagy in TBI. Therapeutic targets that upregulate mitophagy to reduce downstream cascades such as oxidative damage, 
TABLE 2: Summary of abbreviations.

\begin{tabular}{|c|c|}
\hline Abbreviations & Full name \\
\hline IMM & Inner membrane of mitochondrial \\
\hline OMM & Outer membrane of mitochondrial \\
\hline ATP & Adenosine triphosphate \\
\hline $\mathrm{ADP}$ & Adenosine diphosphate \\
\hline OXPHOS & Oxidative phosphorylation \\
\hline ROS & Reactive oxygen species \\
\hline TBI & Traumatic brain injury \\
\hline SOD & Superoxide dismutase \\
\hline PINK1 & PTEN-induced putative kinase 1 \\
\hline NRF2 & Nuclear factor-erythroid 2-related factor 2 \\
\hline KEAP1 & Kelch-like ECH-associated protein 1 \\
\hline TOM & Translocase of the outer membrane \\
\hline OMS & Outer mitochondrial membrane localization signal \\
\hline MFN & Mitochondrial fusion \\
\hline Miro1 & Mitochondrial Rho GTPase 1 \\
\hline VDAC & Voltage-dependent anion channel \\
\hline LIR & LC3 interaction region \\
\hline LC3 & Microtubule-associated protein 1 light chain 3 \\
\hline NIX & NIP3-like protein $\mathrm{X}$ \\
\hline Bnip3 & BCL2/adenovirus E1B $19 \mathrm{kDa}$ interacting protein 3 \\
\hline FUNDC1 & FUN14 domain containing 1 \\
\hline OPA1 & Optic atrophy 1 \\
\hline Ser & Serine \\
\hline Tyr & Tyrosine \\
\hline CK2 & Casein kinase 2 \\
\hline PGAM5 & Phosphoglycerate mutase family member 5 \\
\hline ULK1 & The yeast ATG1 homologues \\
\hline $\mathrm{CL}$ & Cardiolipin \\
\hline PLS & Phospholipid scramblase \\
\hline Thr & Threonine \\
\hline Mdivi-1 & Mitochondrial division inhibitor 1 \\
\hline DCTEIO & 5,6-Dicarboxy-1,1,3,3-tetraethylisoindolin-2-yloxyl \\
\hline T3 & Triiodothyronine \\
\hline NDP52 & Nuclear dot protein $52 \mathrm{kDa}$ \\
\hline TAXBP1 & Tax1-binding protein 1 \\
\hline NIPSNAP1/2 & Nitrophenylphosphatase domain and 147 nonneuronal SNAP25-like protein homolog $1 / 2$ \\
\hline PHB2 & Prohibitin 2 \\
\hline FKBP8 & FKBP Prolyl Isomerase 8 \\
\hline
\end{tabular}

inflammatory response, and cell death may improve neurological dysfunction after TBI. Therefore, we elucidate the mitophagy activators and inhibitors used in TBI in this section (Table 1).

\section{Conclusion}

In recent years, extensive efforts have been made to discover and develop new drugs targeting mitochondrial dysfunction to efficiently rescue or ameliorate the outcomes of traumatic brain injury. In neurons, efficient clearance of damaged mitochondria through mitophagy plays a fundamental role in mitochondrial and metabolic homeostasis, energy supply, neuronal survival, and health. The brain functional outcome was correlated with the severity of the injury after TBI. Therefore, the type, extent, and spatiotemporal distribution of neuronal mitophagy could be related to injury type and severity. Therefore, mitophagy stimulation might be both beneficial and detrimental for tissue homeostasis depending on cellular bioenergetics under certain pathophysiological 
conditions. Despite the extensive studies focusing on the molecular mechanisms that govern mitophagy, some outstanding questions remain. First, it is necessary to further study the interconnected interactions between the different mitophagy pathways and how they coordinate the regulation of mitochondrial removal. Second, the multifaceted roles of mitophagy in cell survival and death are needed to be further investigated. Last, it is necessary to further develop specific, robust, but low-toxicity mitophagy inducers for clinical benefits. Table 2 shows the summary of abbreviations.

\section{Conflicts of Interest}

The authors declare no conflict of interest.

\section{Authors' Contributions}

Mingrui Zhu and Xinqi Huang contributed equally to this work.

\section{Acknowledgments}

This work was supported by the National Natural Science Foundation of China (nos. 82071382 and 81601306); the Priority Academic Program Development of Jiangsu Higher Education Institutions (PAPD); the Jiangsu Maternal and Child Health Research Key Project (F202013); Jiangsu Talent Youth Medical Program (QNRC2016245); Shanghai Key Lab of Forensic Medicine (KF2102); Suzhou Science and Technology Development Project (SYS2020089); and the Fifth Batch of Gusu District Health Talent Training Project (GSWS2019060).

\section{References}

[1] J. R. Friedman and J. Nunnari, "Mitochondrial form and function,” Nature, vol. 505, no. 7483, pp. 335-343, 2014.

[2] Q. Wu, C.-L. Luo, and L.-Y. Tao, "Dynamin-related protein 1 (Drp1) mediating mitophagy contributes to the pathophysiology of nervous system diseases and brain injury," Histology and Histopathology, vol. 32, no. 6, pp. 551-559, 2017.

[3] W. Kuehlbrandt, "Structure and function of mitochondrial membrane protein complexes," BMC Biology, vol. 13, no. 1, p. 89, 2015.

[4] M. E. Raichle and D. A. Gusnard, "Appraising the brain's energy budget," Proceedings of the National Academy of Sciences of the United States of America, vol. 99, no. 16, pp. 10237-10239, 2002.

[5] J. P. Castro, K. Wardelmann, T. Grune, and A. Kleinridders, "Mitochondrial chaperones in the brain: safeguarding brain health and metabolism?," Frontiers in Endocrinology, vol. 9, 2018.

[6] N. S. Swerdlow and H. M. Wilkins, "Mitophagy and the brain," International Journal of Molecular Sciences, vol. 21, no. 24, 2020.

[7] R. J. Youle and A. M. van der Bliek, "Mitochondrial fission, fusion, and stress," Science, vol. 337, no. 6098, pp. 10621065, 2012.

[8] E. F. Fang, Y. Hou, K. Palikaras et al., "Mitophagy inhibits amyloid- $\beta$ and tau pathology and reverses cognitive deficits in models of Alzheimer's disease," Nature Neuroscience, vol. 22, no. 3, pp. 401-412, 2019.
[9] H. Katayama, H. Hama, K. Nagasawa et al., "Visualizing and modulating mitophagy for therapeutic studies of neurodegeneration," Cell, vol. 181, no. 5, pp. 1176-1187.e16, 2020.

[10] J. Ma, H. Ni, Q. Rui et al., "Potential roles of NIX/BNIP3L pathway in rat traumatic brain injury," Cell Transplantation, vol. 28, no. 5, pp. 585-595, 2019.

[11] H. Chao, C. Lin, Q. Zuo et al., "Cardiolipin-dependent mitophagy guides outcome after traumatic brain injury," The Journal of Neuroscience, vol. 39, no. 10, pp. 1930-1943, 2019.

[12] T. P. Ashford and K. R. Porter, "Cytoplasmic components in hepatic cell lysosomes," Journal of Cell Biology, vol. 12, no. 1, p. 198, 1962.

[13] C. L. Campbell and P. E. Thorsness, "Escape of mitochondrial DNA to the nucleus in ymel yeast is mediated by vacuolardependent turnover of abnormal mitochondrial compartments," Journal of Cell Science, vol. 111, pp. 2455-2464, 1998.

[14] J. J. Lemasters, "Selective mitochondrial autophagy, or mitophagy, as a targeted defense against oxidative stress, mitochondrial dysfunction, and aging," Rejuvenation Research, vol. 8, no. 1, pp. 3-5, 2005.

[15] R. L. Schweers, J. Zhang, M. S. Randall et al., "NIX is required for programmed mitochondrial clearance during reticulocyte maturation," Proceedings of the National Academy of Sciences of the United States of America, vol. 104, no. 49, pp. 1950019505, 2007.

[16] D. Narendra, A. Tanaka, D. F. Suen, and R. J. Youle, "Parkin is recruited selectively to impaired mitochondria and promotes their autophagy," Journal of Cell Biology, vol. 183, no. 5, pp. 795-803, 2008.

[17] K. Okamoto, N. Kondo-Okamoto, and Y. Ohsumi, "Mitochondria-anchored receptor Atg32 mediates degradation of mitochondria via selective autophagy," Developmental Cell, vol. 17, no. 1, pp. 87-97, 2009.

[18] L. Liu, D. Feng, G. Chen et al., "Mitochondrial outermembrane protein FUNDC1 mediates hypoxia-induced mitophagy in mammalian cells," Nature Cell Biology, vol. 14, no. 2, pp. 177-185, 2012.

[19] S. Melser, E. H. Chatelain, J. Lavie et al., "Rheb regulates mitophagy induced by mitochondrial energetic status," Cell Metabolism, vol. 17, no. 5, pp. 719-730, 2013.

[20] C. T. Chu, J. Ji, R. K. Dagda et al., "Cardiolipin externalization to the outer mitochondrial membrane acts as an elimination signal for mitophagy in neuronal cells," Nature Cell Biology, vol. 15, no. 10, pp. 1197-1205, 2013.

[21] Y. Wang, N. Liu, and B. Lu, "Mechanisms and roles of mitophagy in neurodegenerative diseases," CNS Neuroscience \& Therapeutics, vol. 25, no. 7, pp. 859-875, 2019.

[22] H. Murata, H. Takamatsu, S. Liu, K. Kataoka, N. H. Huh, and M. Sakaguchi, "NRF2 regulates PINK1 expression under oxidative stress conditions," PLoS One, vol. 10, no. 11, 2015.

[23] S. Rikka, M. N. Quinsay, R. L. Thomas et al., "Bnip3 impairs mitochondrial bioenergetics and stimulates mitochondrial turnover," Cell Death and Differentiation, vol. 18, no. 4, pp. 721-731, 2011.

[24] P. Terešak, A. Lapao, N. Subic, P. Boya, Z. Elazar, and A. Simonsen, "Regulation of PRKN-independent mitophagy," Autophagy, vol. 25, pp. 1-16, 2021.

[25] E. M. Valente, S. Salvi, T. Ialongo et al., "PINK1 mutations are associated with sporadic early-onset parkinsonism," Annals of Neurology, vol. 56, no. 3, pp. 336-341, 2004. 
[26] N. Matsuda, S. Sato, K. Shiba et al., "PINK1 stabilized by mitochondrial depolarization recruits Parkin to damaged mitochondria and activates latent Parkin for mitophagy," Journal of Cell Biology, vol. 189, no. 2, pp. 211-221, 2010.

[27] Q. Wu, C. Gao, H. Wang et al., "Mdivi-1 alleviates blood-brain barrier disruption and cell death in experimental traumatic brain injury by mitigating autophagy dysfunction and mitophagy activation," The International Journal of Biochemistry \& Cell Biology, vol. 94, pp. 44-55, 2018.

[28] Y. Chen, K. Gong, Q. Xu et al., "Phosphoglycerate mutase 5 knockdown alleviates neuronal injury after traumatic brain injury through Drp1-mediated mitochondrial dysfunction," Antioxidants \& Redox Signaling, vol. 34, no. 2, pp. 154-170, 2021.

[29] Y. Z. Ren, B. Z. Zhang, X. J. Zhao, and Z. Y. Zhang, "Resolvin D1 ameliorates cognitive impairment following traumatic brain injury via protecting astrocytic mitochondria," Journal of Neurochemistry, vol. 154, no. 5, pp. 530-546, 2020.

[30] C. Lin, N. Li, H. Chang et al., "Dual effects of thyroid hormone on neurons and neurogenesis in traumatic brain injury," Cell Death \& Disease, vol. 11, no. 8, p. 671, 2020.

[31] Y. Chen, J. Deng, P. Wang et al., "PINK1 and Parkin are genetic modifiers for FUS-induced neurodegeneration," Human Molecular Genetics, vol. 25, no. 23, pp. 5059-5068, 2016.

[32] Y. Wen, Y. Gu, X. Tang, and Z. Hu, "PINK1 overexpression protects against cerebral ischemia through Parkin regulation," Environmental Toxicology, vol. 35, no. 2, pp. 188-193, 2020.

[33] M. Onishi, K. Yamano, M. Sato, N. Matsuda, and K. Okamoto, "Molecular mechanisms and physiological functions of mitophagy," EMBO Journal, vol. 40, no. 3, 2021.

[34] Y. Xie, J. Liu, R. Kang, and D. Tang, "Mitophagy receptors in tumor biology," Frontiers in Cell and Development Biology, vol. 8, article 594203, 2020.

[35] Y. Wei, W. C. Chiang, R. Sumpter Jr., P. Mishra, and B. Levine, "Prohibitin 2 is an inner mitochondrial membrane mitophagy receptor," Cell, vol. 168, no. 1-2, pp. 224-238.e10, 2017.

[36] Y. Rui, K. Ke, L. Li et al., "Up-regulated expression of Bnip3L after intracerebral hemorrhage in adult rats," Journal of Molecular Histology, vol. 44, no. 5, pp. 497-505, 2013.

[37] Y. Yuan, Y. Zheng, X. Zhang et al., "BNIP3L/NIX-mediated mitophagy protects against ischemic brain injury independent of PARK2," Autophagy, vol. 13, no. 10, pp. 17541766, 2017.

[38] K. M. Regula, K. Ens, and L. A. Kirshenbaum, "Inducible expression of BNIP3 provokes mitochondrial defects and hypoxia-mediated cell death of ventricular myocytes," Circulation Research, vol. 91, no. 3, pp. 226-231, 2002.

[39] A. Hamacher-Brady, S. C. Choe, J. Krijnse-Locker, and N. R. Brady, "Intramitochondrial recruitment of endolysosomes mediates Smac degradation and constitutes a novel intrinsic apoptosis antagonizing function of XIAP E3 ligase," Cell Death and Differentiation, vol. 21, no. 12, pp. 1862-1876, 2014.

[40] A. M. Lamade, T. S. Anthonymuthu, Z. E. Hier, Y. Gao, V. E. Kagan, and H. Bayır, "Mitochondrial damage \& lipid signaling in traumatic brain injury," Experimental Neurology, vol. 329, 2020.

[41] S. Kalgudi and K. M. Ho, "Incidence of antithrombin deficiency and anti-cardiolipin antibodies after severe traumatic brain injury: a prospective cohort study," Neurocritical Care, vol. 34, no. 1, pp. 227-235, 2021.
[42] M. M. Saw, J. Chamberlain, M. Barr, M. P. Morgan, J. R. Burnett, and K. M. Ho, "Differential disruption of blood-brain barrier in severe traumatic brain injury," Neurocritical Care, vol. 20, no. 2, pp. 209-216, 2014.

[43] H. Chao, T. S. Anthonymuthu, E. M. Kenny et al., "Disentangling oxidation/hydrolysis reactions of brain mitochondrial cardiolipins in pathogenesis of traumatic injury," Insight, vol. 3, no. 21, 2018.

[44] C. T. Chu, H. Bayir, and V. E. Kagan, "LC3 binds externalized cardiolipin on injured mitochondria to signal mitophagy in neurons implications for Parkinson disease," Autophagy, vol. 10, no. 2, pp. 376-378, 2014.

[45] T. Ryan, V. V. Bamm, M. G. Stykel et al., "Cardiolipin exposure on the outer mitochondrial membrane modulates $\alpha$-synuclein,” Nature Communications, vol. 9, no. 1, p. 817, 2018.

[46] S. Melser, J. Lavie, and G. Benard, "Mitochondrial degradation and energy metabolism," Biochimica et Biophysica ActaMolecular Cell Research, vol. 1853, no. 10, pp. 2812-2821, 2015.

[47] D. Ma, X. Bai, S. Guo, and Y. Jiang, "The switch I region of Rheb is critical for its interaction with FKBP38," The Journal of Biological Chemistry, vol. 283, no. 38, pp. 25963-25970, 2008.

[48] W. Yang, D. Pang, M. Chen et al., "Rheb mediates neuronalactivity-induced mitochondrial energetics through mTORC1-independent PDH activation," Developmental Cell, vol. 56, no. 6, pp. 811-825.e6, 2021.

[49] S. Han, M. Zhang, Y. Y. Jeong, D. J. Margolis, and Q. Cai, "The role of mitophagy in the regulation of mitochondrial energetic status in neurons," Autophagy, vol. 17, no. 12, pp. 4182-4201, 2021.

[50] S. Han, Y. Y. Jeong, P. Sheshadri, X. Su, and Q. Cai, "Mitophagy regulates integrity of mitochondria at synapses and is critical for synaptic maintenance," EMBO Reports, vol. 21, no. 9, article e49801, 2020.

[51] J. E. Bailes and C. V. Borlongan, "Traumatic brain injury," CNS Neuroscience \& Therapeutics, vol. 26, no. 6, pp. 593-594, 2020.

[52] A. D. Filev, D. N. Silachev, I. A. Ryzhkov et al., "Effect of xenon treatment on gene expression in brain tissue after traumatic brain injury in rats," Brain Sciences, vol. 11, no. 7, 2021.

[53] P. Maiti, S. Peruzzaro, N. Kolli et al., “Transplantation of mesenchymal stem cells overexpressing interleukin-10 induces autophagy response and promotes neuroprotection in a rat model of TBI," Journal of Cellular and Molecular Medicine, vol. 23, no. 8, pp. 5211-5224, 2019.

[54] L.-Y. Yang, N. H. Greig, D. Tweedie et al., "The p53 inactivators pifithrin- $\mu$ and pifithrin- $\alpha$ mitigate TBI-induced neuronal damage through regulation of oxidative stress, neuroinflammation, autophagy and mitophagy," Experimental Neurology, vol. 324, article 113135, 2020.

[55] Y. Chen, J. Meng, Q. Xu et al., "Rapamycin improves the neuroprotection effect of inhibition of NLRP3 inflammasome activation after TBI," Brain Research, vol. 1710, pp. 163-172, 2019.

[56] C. Lin, H. Chao, Z. Li et al., "Melatonin attenuates traumatic brain injury-induced inflammation: a possible role for mitophagy," Journal of Pineal Research, vol. 61, no. 2, pp. 177-186, 2016.

[57] H. Ding, Y. Li, S. Chen et al., "Fisetin ameliorates cognitive impairment by activating mitophagy and suppressing neuroinflammation in rats with sepsis-associated encephalopathy," CNS Neuroscience \& Therapeutics, vol. 28, no. 2, 2022. 
[58] S. Zheng, D. Jian, H. Gan, L. Wang, J. Zhao, and X. Zhai, "FUNDC1 inhibits NLRP3-mediated inflammation after intracerebral hemorrhage by promoting mitophagy in mice," Neuroscience Letters, vol. 756, 2021.

[59] K. Okamoto, "Organellophagy: eliminating cellular building blocks via selective autophagy," Journal of Cell Biology, vol. 205, no. 4, pp. 435-445, 2014.

[60] Y. Liu, Z. Bao, X. Xu et al., "Extracellular signal-regulated kinase/nuclear factor-erythroid2-like2/heme oxygenase-1 pathway-mediated mitophagy alleviates traumatic brain injury-induced intestinal mucosa damage and epithelial barrier dysfunction," Journal of Neurotrauma, vol. 34, no. 13, pp. 2119-2131, 2017.

[61] R. J. Youle and D. P. Narendra, "Mechanisms of mitophagy," Nature Reviews Molecular Cell Biology, vol. 12, no. 1, pp. 914, 2011.

[62] C. Fleury, B. Mignotte, and J. L. Vayssiere, "Mitochondrial reactive oxygen species in cell death signaling," Biochimie, vol. 84, no. 2-3, pp. 131-141, 2002.

[63] I. Kim, S. Rodriguez-Enriquez, and J. J. Lemasters, "Selective degradation of mitochondria by mitophagy," Archives of Biochemistry and Biophysics, vol. 462, no. 2, pp. 245-253, 2007.

[64] F. Niu, J. Dong, X. Xu, B. Zhang, and B. Liu, "Mitochondrial division inhibitor 1 prevents early-stage induction of mitophagy and accelerated cell death in a rat model of moderate controlled cortical impact brain injury," World Neurosurgery, vol. 122, pp. E1090-E1101, 2019.

[65] T. Saito and J. Sadoshima, "Molecular mechanisms of mitochondrial autophagy/mitophagy in the heart," Circulation Research, vol. 116, no. 8, pp. 1477-1490, 2015.

[66] R. Zakarya, A. Sapkota, Y. L. Chan et al., "Nitroxides affect neurological deficits and lesion size induced by a rat model of traumatic brain injury," Nitric Oxide-Biology and Chemistry, vol. 97, pp. 57-65, 2020.

[67] H. Chi, H. Y. Chang, and T. K. Sang, "Neuronal cell death mechanisms in major neurodegenerative diseases," International Journal of Molecular Sciences, vol. 19, no. 10, 2018.

[68] P. M. Lenzlinger, M. C. Morganti-Kossmann, H. L. Laurer, and M. I. TK, "The duality of the inflammatory response to traumatic brain injury," Molecular Neurobiology, vol. 24, no. 1-3, pp. 169-181, 2001.

[69] X. Zhang, Y. Chen, L. W. Jenkins, P. M. Kochanek, and R. S. Clark, "Bench-to-bedside review: apoptosis/programmed cell death triggered by traumatic brain injury," Critical Care, vol. 9, no. 1, pp. 66-75, 2005.

[70] D. Tang, R. Kang, T. V. Berghe, P. Vandenabeele, and G. Kroemer, "The molecular machinery of regulated cell death," Cell Research, vol. 29, no. 5, pp. 347-364, 2019.

[71] K. Ma, G. Chen, W. Li, O. Kepp, Y. Zhu, and Q. Chen, "Mitophagy, mitochondrial homeostasis, and cell fate," Frontiers in Cell and Development Biology, vol. 8, p. 467, 2020.

[72] S. Li, J. Zhang, C. Liu et al., "The role of mitophagy in regulating cell death," Oxidative Medicine and Cellular Longevity, vol. 2021, Article ID 6617256, 12 pages, 2021.

[73] T. Bergsbaken, S. L. Fink, and B. T. Cookson, "Pyroptosis: host cell death and inflammation," Nature Reviews. Microbiology, vol. 7, no. 2, pp. 99-109, 2009.

[74] H. G. Ding, Y. Li, X. S. Li et al., "Hypercapnia promotes microglial pyroptosis via inhibiting mitophagy in hypoxemic adult rats," CNS Neuroscience \& Therapeutics, vol. 26, no. 11, pp. 1134-1146, 2020.
[75] Z. Hu, Y. Yuan, X. Zhang et al., "Human umbilical cord mesenchymal stem cell-derived exosomes attenuate oxygenglucose deprivation/reperfusion-induced microglial pyroptosis by promoting FOXO3a-dependent mitophagy," Oxidative Medicine and Cellular Longevity, vol. 2021, Article ID 6219715, 2021.

[76] X. Han, T. Xu, Q. Fang et al., "Quercetin hinders microglial activation to alleviate neurotoxicity via the interplay between NLRP3 inflammasome and mitophagy," Redox Biology, vol. 44, 2021.

[77] J. Li, F. Cao, H. L. Yin et al., "Ferroptosis: past, present and future," Cell Death \& Disease, vol. 11, no. 2, p. 88, 2020.

[78] X. Wang, H. Ma, J. Sun et al., "Mitochondrial ferritin deficiency promotes osteoblastic ferroptosis via mitophagy in type 2 diabetic osteoporosis," Biological Trace Element Research, vol. 200, no. 1, pp. 298-307, 2022. 